\title{
Sirtuin 1 Modulation in Rat Model of Acetaminophen-Induced Hepatotoxicity
}

\author{
L. WOJNAROVÁ ${ }^{1}$, N. KUTINOVÁ CANOVÁ ${ }^{1}$, H. FARGHALI ${ }^{1}$, T. KUČERA ${ }^{2}$ \\ ${ }^{1}$ Institute of Pharmacology, First Faculty of Medicine, Charles University, Prague, Czech Republic, \\ ${ }^{2}$ Institute of Histology and Embryology, First Faculty of Medicine, Charles University, Prague, \\ Czech Republic
}

Received September 15, 2015

Accepted September 25, 2015

\section{Summary}

Sirtuin 1 (SIRT1) is involved in important biological processes such as energy metabolism and regulatory functions of the cell cycle, apoptosis, and inflammation. Our previous studies have shown hepatoprotective effect of polyphenolic compound resveratrol, which is also an activator of SIRT1. Therefore, the aim of our present study was to clarify the role of SIRT1 in process of hepatoprotection in animal model of drug-induced liver damage. Male Wistar rats were used for both in vivo and in vitro studies. Hepatotoxicity was induced by single dose of acetaminophen (APAP). Some rats and hepatocytes were treated by resveratrol or synthetic selective activator of sirtuin 1 (CAY10591). The degree of hepatotoxicity, the activity and expression of the SIRT1 were determined by biochemical, histological and molecular-biological assessments of gained samples (plasma, liver tissue, culture media and hepatocytes). Resveratrol and CAY attenuated APAP-induced hepatotoxicity in vivo and in vitro. Moreover, both drugs enhanced APAPreduced SIRT1 activity. Our results show that modulation of the SIRT1 activity plays a role in hepatoprotection. Synthetic activators of SIRT1 would help in understanding the role of SIRT1 and are therefore a major boost towards the search for specific treatment of liver disease.

\section{Key words}

SIRT1 • Resveratrol • Acetaminophen • CAY10591 • Liver

\section{Corresponding author}

L. Wojnarová, Institute of Pharmacology, First Faculty of Medicine, Charles University, Albertov 4, 12800 Prague 2, Czech Republic. E-mail: lea.wojnarova@lf1.cuni.cz

\section{Introduction}

Drug-induced liver injury (DILI) is the most frequent cause of hepatic dysfunction and it is the most common cause of acute liver failure in the United States. Over 1000 medications and herbal products have been associated in the development of DILI. In many studies, acetaminophen-induced liver injury is commonly used as a standard hepatotoxicity model in rodents to test the hepatoprotective effects of herbal and other compounds. Acetaminophen (APAP, paracetamol) belongs to the most widely used analgetic and antipyretic drugs. Overdose can cause hepatotoxicity in experimental animals and humans (Brune et al. 2015, Ingawale et al. 2014, Jaeschke et al. 2013, McGill et al. 2012). But what is a maximum safe dose of APAP? The safety of this drug and its toxic reactions are influenced by a large number of factors, both known and unknown (Kaplowitz et al. 2004). It is well established that APAP is detoxified by three major pathways. The majority of APAP dose is conjugated with glucuronate and sulfate and remaining part of APAP especially in its excess is metabolized by an alternative pathway known as cytochrome $\mathrm{P} 450$ oxidase enzyme system (mainly CYP2E1). The latter metabolic pathway leads to the formation of a reactive metabolite, N-acetyl-p-benzoquinone imine (NAPQI). NAPQI is detoxified especially by glutathione (GSH) to form APAP-SG conjugate (Kučera et al. 2011, Roušar et al. 2009). In the case of GSH depletion, this metabolite covalently binds to cysteine groups on protein, forming APAP-protein adducts in the cell and in mitochondria (Jaeschke et al. 2013, Wang et al. 2015). It results in 
conformational and functional changes in cellular proteins, increased reactive oxygen and reactive nitrogen species production (ROS, RNS), lipoperoxidation, mitochondrial permeability and transition pore opening. These are key events leading to cell death (Bajt et al. 2006, Du et al. 2015, Jaeschke et al. 2013, Karthivashan et al. 2015, Roušar et al. 2009). Despite the processes mentioned above, the detailed understanding of the mechanism of APAP toxic effect still remains unknown (Kaplowitz et al. 2004, Roušar et al. 2009).

Our previous studies have shown hepatoprotective effect of resveratrol in lipopolysaccharide/D-Galactosamine (LPS/D-GAIN) model of hepatotoxicity (Černý et al. 2009, Farghali et al. 2014). Resveratrol pretreatment led to the overall improvement in hepatotoxic markers and morphology after the hepatic insult. Several studies have also highlighted the hepatoprotective properties of resveratrol (Bishayee et al. 2010). Resveratrol (trans-3,4',5-trihydroxystilbene) is a polyphenolic compound found in a plant sources such as peanuts, grapes and red wine and it's one of the extensively studied natural product, with wide ranging biological activity and clinical potentials. Many studies have suggested that resveratrol possess many pharmacological actions including anti-aging, anticarcinogenic, anti-inflammatory, and antioxidant properties (Wang et al. 2015). Moreover, resveratrol is also the most widely investigated activator of sirtuin 1 (SIRT1, silent information regulator T1) (Baur 2010, Kutinová Canová et al. 2012, Ruderman et al. 2010, Silva and Wahlestedt 2010).

SIRT1 is the evolutionarily conserved $\mathrm{NAD}^{+}$-dependent histone/protein deacetylase whose function is linked to cellular metabolism. It plays a role in metabolism of numerous tissues, including liver, muscle, adipose tissue, heart, and endothelium (Chang and Guarente 2014). Moreover, an important target for SIRT1 is nuclear factor-kappa $\mathrm{B}(\mathrm{NF}-\kappa \mathrm{B})$, a regulator of many processes, including the cell cycle, proliferation, apoptosis, and inflammation (Yang et al. 2007). Several small-molecule SIRT1 activators, among others e.g. CAY1059 and SRT1720, were synthesized with higher pharmacologic potency compared with the traditionally studied SIRT1 activator resveratrol (Feige et al. 2008, Nayagam et al. 2006).

The main goal of the present study was to clarify the role of SIRT1 in the process of hepatotoxicity/ hepatoprotection in animal model of APAP-induced acute liver injury and to investigate SIRT1 activation and protein expression under treatment with natural SIRT1 activator resveratrol and selective synthetic SIRT1 activator CAY10591 (CAY) both in vivo and in vitro.

\section{Materials and Methods}

\section{Materials}

CAY10591 was obtained from Cayman Pharma (Neratovice, Czech Republic) and collagenase type IV from Sevapharma (Prague, Czech Republic). Bio-Rad protein DC assay dye reagent was from Bio-Rad (Prague, Czech Republic). Acetaminophen, resveratrol (3,4',5trihydroxy-trans-stilbene, 5-[(1E)-2-(4-hydroxyphenyl) ethenyl]-1,3-benzenediol $\geq 99 \%$ GC), SIRT1 assay kit, PEG400, saline, tris-HCl, Nonidet P40, dimethyl sulfoxide (DMSO), isopropyl alcohol, Tween 20, 2-thiobarbituric acid, tetraethoxypropane, trichloroacetic acid, sodium dodecyl sulphate, ammonium persulfate,

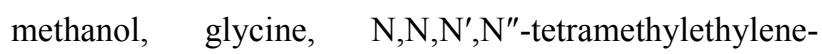
diamine, $\mathrm{NaCl}, \mathrm{KCl}, \mathrm{Na}_{2} \mathrm{HPO}_{4}, \mathrm{KH}_{2} \mathrm{PO}_{4}$, filter paper, nitrocellulose membrane, anti-mouse IgG (whole molecule) peroxidase antibody and mouse monoclonal anti-beta-actin antibody, protease and phosphatase inhibitor cocktails, and other standard chemicals were purchased from Sigma-Aldrich (Prague, Czech Republic). SirT1 (1F3) mouse mAb antibody was from Cell Signaling Technology through BioTech (Prague, Czech Republic). Non-fat dry milk was also from BioTech. Water for injection $100 \% \mathrm{w} / \mathrm{v}$ was obtained from Baxter (Czech Republic, Prague).

\section{Animals}

Outbred male Wistar rats (Velaz-Lysolaje, Czech Republic) of 200-350 g body weight were used throughout the present studies. Rats were allowed to tap water and standard granulated diet ad libitum and were maintained under standard light (12/12h light/dark), temperature $\left(22 \pm 2{ }^{\circ} \mathrm{C}\right)$ and relative humidity $(50 \pm 10 \%)$ conditions. All rats received humane care in compliance with the general guidelines of the First Faculty of Medicine, Charles University in Prague. The study protocol was approved by the Faculty Ethical Committee.

\section{Drug treatments}

The rats were divided randomly into five groups of six animals each and treated as follows: 1. Control polyethyleneglycol $400(40 \%)+$ DMSO, 2. CAY - CAY $0.5 \mathrm{mg} / \mathrm{kg}$ in DMSO, 3. APAP - acetaminophen $1 \mathrm{~g} / \mathrm{kg}$ in PEG 400 (40\%), 4. APAP+CAY - acetaminophen $1 \mathrm{~g} / \mathrm{kg}$ 
in PEG $400(40 \%)+\mathrm{CAY} 0.5 \mathrm{mg} / \mathrm{kg}$ in DMSO, 5. APAP+RES - acetaminophen $1 \mathrm{~g} / \mathrm{kg}$ in PEG 400 $(40 \%)+$ RES $30 \mathrm{mg} / \mathrm{kg}$ in DMSO.

To induce acute liver injury in rats, $1 \mathrm{~g} / \mathrm{kg}$ of APAP was injected intraperitoneally from a $0.2 \mathrm{~g} / \mathrm{ml}$ solution in $40 \%$ PEG 400 (PEG 400 in saline). It was followed after one hour by intraperitoneal injection of CAY10591 or resveratrol, both dissolved in DMSO of total volume of $1.5 \mathrm{ml} / \mathrm{kg}$. Twenty-four hours after APAP application, the animals were anesthetized with diethylether and then euthanized by exsanguination. Blood samples were collected into heparinized tubes. Plasma was immediately isolated by centrifugation at 4,000 rpm for $10 \mathrm{~min}$ and used for assessment of alanine aminotransferase (ALT), total bilirubin and nitric oxide (NO) as nitrite $\left(\mathrm{NO}_{2}{ }^{-}\right)$levels. Their liver samples were immediately homogenized in cooled Tris-HCl buffer or snap frozen in liquid nitrogen for Western blot analysis. The liver homogenates were used for determination of total lipid peroxidation assessed as thiobarbituric acid reactive substances (TBARS) and conjugated dienes (CD).

\section{Isolation and culture of primary rat hepatocytes}

Hepatocytes were isolated from untreated rats using the standard two phase collagenase perfusion method (Berry et al. 1991). Separated hepatocytes were counted and cell viability was assessed by trypan blue exclusion method. The hepatocyte cell viability was greater than $85 \%$. Cells were seeded on collagen-coated polystyrene Nunclon ${ }^{\mathrm{TM}}$ dishes at density of 104000 viable cells $/ \mathrm{cm}^{2}$. They were incubated in complete medium (William's medium E, penicillin/streptomycin $1 \%$, glutamine $1 \%$, insulin $0.06 \%$, FBS-fetal bovine serum $5 \%$ ) at $37{ }^{\circ} \mathrm{C}$ in a humid atmosphere with $5 \%$ $\mathrm{CO}_{2}$ throughout the study. Unattached hepatocytes were removed $3 \mathrm{~h}$ after seeding and remaining hepatocytes further cultured in fresh complete medium overnight. Hepatocytes were then treated with fresh medium alone or with acetaminophen (APAP, $5 \mathrm{mM}$ ). Thirty minutes later, vehicle with DMSO ( $0.1 \%$ of final concentration), resveratrol (RES, $20 \mu \mathrm{M}$ ) or CAY10591 (CAY, $30 \mu \mathrm{M}$ ) were added to respective hepatocyte cultures. After $24 \mathrm{~h}$, medium samples were collected for biochemical analysis and hepatocyte viability was assessed by MTT test. At the end of experiments, some cultured hepatocytes were washed by cooled phosphate buffered saline and lysed in RIPA buffer containing protease inhibitor cocktail. The homogenates were centrifuged at $14,000 \mathrm{~g}$ for $10 \mathrm{~min}$ at $4{ }^{\circ} \mathrm{C}$ and thereafter used for SIRT 1 activity measurement.

\section{MTT/cell viability test}

MTT (3-[4,5-dimethylthiazol-2-yl]-2,5-diphenyltetrazolium bromide) was used both to assess the optimal non-toxic concentration of drugs suitable for our study and to measure hepatocyte viability at the end of in vitro experiments following test description by Kutinova Canova et al. (2008).

\section{Biochemical analysis}

ALT and bilirubin concentrations in the medium or plasma samples were measured using customized diagnostic kits according manufacturer's instruction (Vian Diagnostics, Prague, Czech Republic).

Medium $\mathrm{NO}_{2}^{-}$, the stable end-product of $\mathrm{NO}$ oxidation, was determined spectrofotometrically by using Griess reagent (1\% sulfanilamide, $0.1 \%$ naphtylethylendiamine, $2.5 \%$ trihydrogenphosphoric acid). The absorbance at $540 \mathrm{~nm}$ was recorded and the $\mathrm{NO}_{2}^{-}$levels were calculated from $\mathrm{NaNO}_{2}$ standard curve.

\section{Evaluation of oxidative stress parameters}

The measurement of homogenate levels of TBARS and conjugated dienes were carried out according to Farghali et al. (2009).

\section{SIRT1 deacetylase activity assay}

SIRT1 deacetylase activity was evaluated in $5 \mu 1$ of the whole liver lysate as well as cultured hepatocyte lysate according to manual instruction of fluorometric SIRT1 Assay Kit (Sigma-Aldrich). The measured fluorescence was directly proportional to deacetylation activity of the SIRT1 enzyme in the sample. All measurements were performed in duplicate and the results were reported as arbitrary units of relative fluorescence per $1 \mathrm{mg}$ of lysate protein (assessed by Bio-Rad protein DC assay).

\section{Histological evaluation}

After the excised liver $\left(1 \mathrm{~cm}^{3}\right)$ fixation in $4 \%$ paraformaldehyde in $\mathrm{PBS}$, thin tissue paraffin sections $(5 \mu \mathrm{m})$ were cut by microtome, stained with hematoxylin and eosin and examined by light microscope.

\section{Immunoblotting}

Isolated liver samples were lysed with RIPA buffer (2 M TRIS, $5 \mathrm{M} \mathrm{NaCl}, 0.5 \mathrm{M}$ EDTA, NP-40, NAF) and homogenized with an electric homogenizer. The samples were then centrifuged for $20 \mathrm{~min}$ at $12,000 \mathrm{rpm}$ at $4{ }^{\circ} \mathrm{C}$ and supernatant was collected. The 
cell lysates were mixed (1:1) with sample buffer $(2 \mathrm{x}$ Laemmli buffer $950 \mu 1+50 \mu \mathrm{l}$ of $\beta$-mercaptoethanol) and then heated for $10 \mathrm{~min}$ at $90{ }^{\circ} \mathrm{C}$. Proteins (assessed by Bio-Rad protein DC assay) from the tissue samples were separated on $10 \%$ SDS-acrylamide gel and transferred to a nitrocellulose membrane by electrophoresis overnight at $4{ }^{\circ} \mathrm{C}$. Membranes were blocked for $2 \mathrm{~h}$ with $5 \%$ non-fat milk in Tris-buffered solution, at $4{ }^{\circ} \mathrm{C}$. Membranes were then washed in a washing buffer $\left(\mathrm{NaCl}, \mathrm{KCl}, \mathrm{Na}_{2} \mathrm{HPO}_{4}\right.$, $\mathrm{KH}_{2} \mathrm{PO}_{4}$, Tween, $\mathrm{H}_{2} \mathrm{O}$ ). They were then incubated with mouse primary antibody against SIRT1 $(1: 1,000)$ or betaactin primary antibody $(1: 5,000)$ and followed with corresponding secondary rabbit antibody anti-mouse IgG HRP conjugate $(1: 80,000)$. For visualization was used chemiluminescence labeling with Super Signal West Pico Chemiluminescent Substrate (GeneTiCA, Prague, Czech Republic). Bands were detected with the use Molecular Imager VersaDoc ${ }^{\mathrm{TM}}$ MP 5000 System and analyzed by Quantity One 1-D Analysis Software (Bio-Rad, Prague, Czech Republic). Optical densities of SIRT1 and betaactin bands were normalized by the corresponding loading control and then to the mean of the corresponding control group (Lekić et al. 2013).

a)

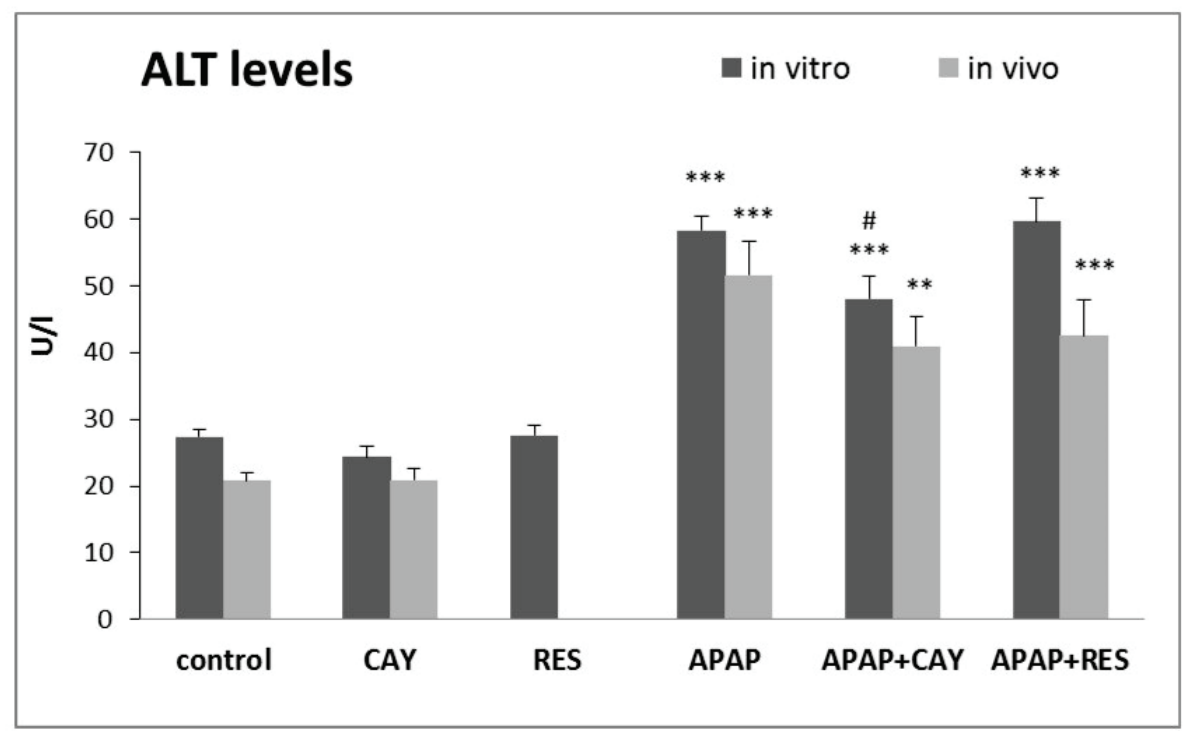

b)

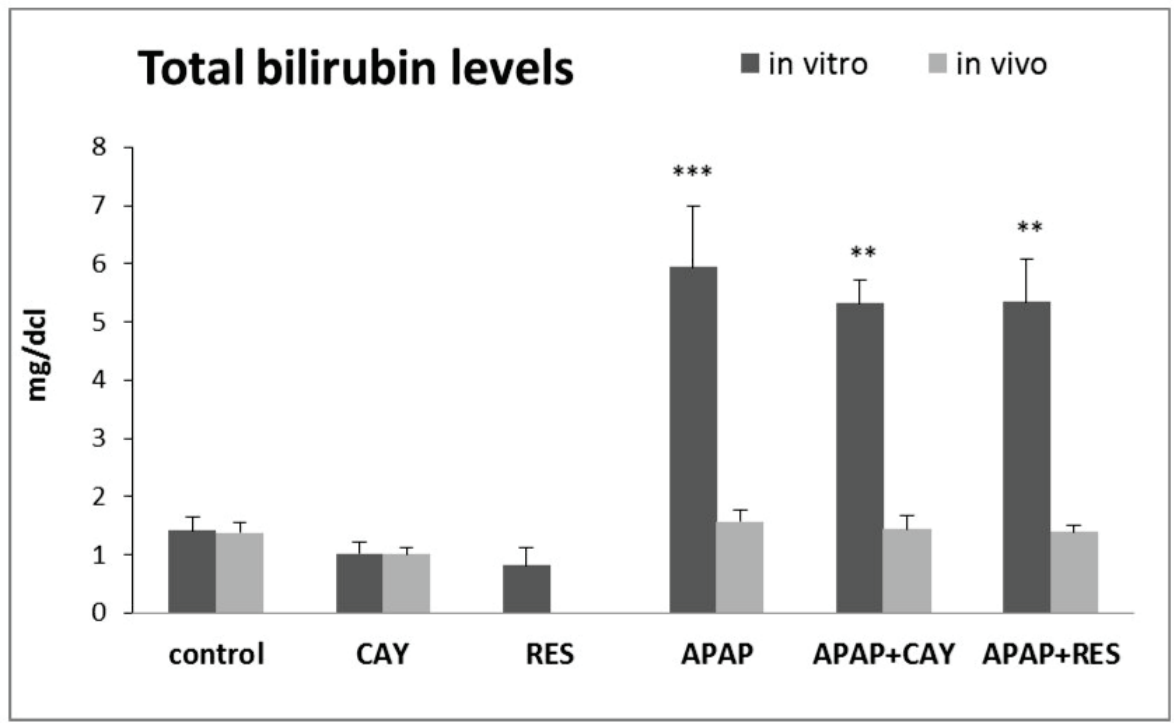

Fig. 1. Effect of specific SIRT1 activator, CAY10591, and resveratrol treatments on hepatocyte function in acute APAP-induced hepatocyte/liver injury in vitro and in vivo expressed as medium or plasma levels of a) Alanine aminotransferase ALT and b) Bilirubin. Control (24 hour-vehicle treated hepatocytes or rats); CAY (CAY10591: $30 \mu \mathrm{M}$ in vitro, $0.5 \mathrm{mg} / \mathrm{kg}$ in vivo); RES (Resveratrol: $20 \mu \mathrm{M}$ in vitro, $30 \mathrm{mg} / \mathrm{kg}$ in vivo); APAP (Acetaminophen: $5 \mathrm{mM}$ in vitro, $1 \mathrm{~g} / \mathrm{kg}$ in vivo); APAP + CAY (combination of Acetaminophen and CAY10591 in the stated doses); APAP + RES (combination of Acetaminophen and Resveratrol in the stated doses). Data are expressed as means $\pm \operatorname{SEM}(n=6)$ : ** $\mathrm{P}<0.01, * * * \mathrm{P}<0.001$ vs. respective control; \# $\mathrm{P}<0.05$ vs. APAP in vitro. 


\section{Statistical analysis}

The statistical significance of differences of mean scores was determined using one-way analysis of variance (ANOVA) followed by Bonferroni Multiple Comparisons test (Graph-Pad Prism 4.03, Graph Pad Software, San Diego, CA, USA). P-value less than 0.05 was considered to be statistically significant. Data were expressed as means \pm SEM (standard error of mean). All experiments were performed in means of 6 animals per group for in vivo experiments and 6 hepatocyte cultures per group of 3 independent in vitro experiments.

\section{Results}

Effects of CAY in comparison with RES treatment on liver function in APAP treated rats in vitro and in vivo

We measured the levels of ALT and bilirubin in plasma to detect the extent of hepatotoxicity (Fig. 1a). The APAP treatment in rats produced significant two-fold increase of ALT release $(\mathrm{P}<0.001)$ compared to the control group both in vitro and in vivo. Moreover, treatment with RES and CAY after induction of hepatotoxicity significantly lowered the ALT parameters in vivo. Interesting difference was in in vitro experiments, where only CAY treatment slightly reduced ALT, whereas RES did not influence the resulting values. Significant increase of bilirubin, fivefold higher, was observed in hepatocyte cultures compared to the control. There was a tendency of resveratrol and CAY to reduce APAP-increased total bilirubin levels, both in vitro and in vivo (Fig. 1b).

Effects of CAY in comparison with RES treatment on $\mathrm{NO}_{2}{ }^{-}$in vitro and in vivo and oxidative stress parameters in APAP treated rats in vivo

Figures $2 \mathrm{a}$ and $2 \mathrm{~b}$ demonstrate that APAP treatment produced moderate increase of lipid peroxidation as evidenced by the formation of TBARS and conjugated dienes (CD), respectively, as a result of oxidative stress in liver. Single APAP treatment significantly increased both TBARS and $\mathrm{CD}(\mathrm{P}<0.05)$ in homogenate. CAY following APAP treatment slightly reduced only $\mathrm{CD}$ levels in contrast with RES, which reduced the levels of both markers. Moreover, APAP significantly increased medium $\mathrm{NO}_{2}^{-}$levels $(\mathrm{P}<0.001)$ in vitro. On the other hand, $\mathrm{NO}_{2}{ }^{-}$plasma levels were not significantly affected by any treatment even though CAY and resveratrol slightly reduced it (Fig. 2c).

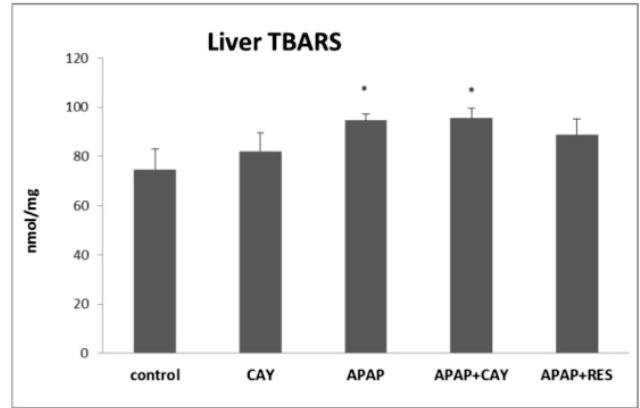

b)

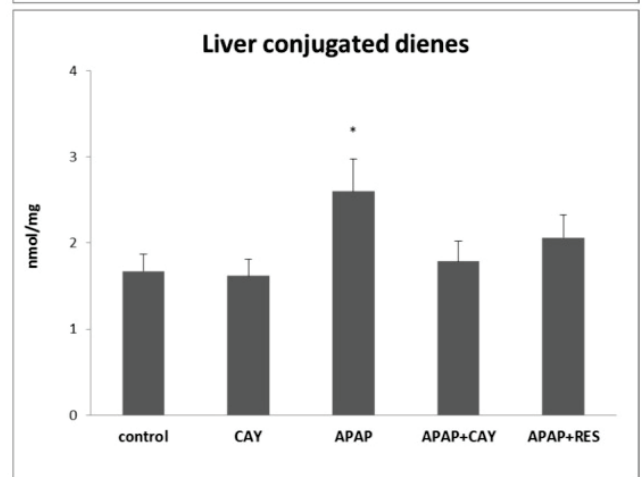

c)

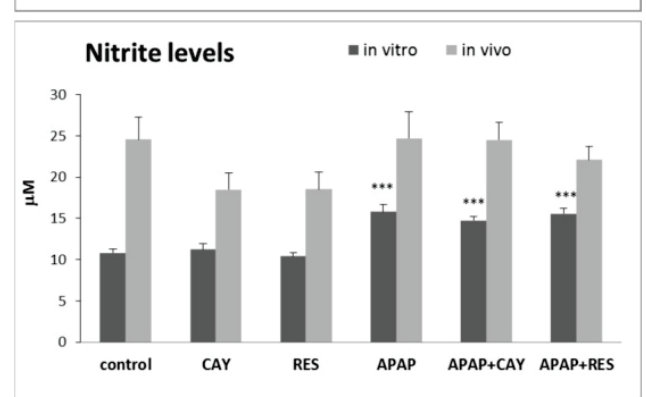

Fig. 2. Effect of specific SIRT1 activator, CAY10591 in comparison with resveratrol treatment in APAP-induced hepatocyte/liver injury on the formation of a) Thiobarbituric acid reactive substances (TBARS) and b) Conjugated dienes (CD) in vivo, and c) $\mathrm{NO}_{2}^{-}$production in vitro and in vivo. Control (24 hour-vehicle treated hepatocytes or rats); CAY (CAY10591: $30 \mu \mathrm{M}$ in vitro, $0.5 \mathrm{mg} / \mathrm{kg}$ in vivo); RES (Resveratrol: $20 \mu \mathrm{M}$ in vitro, $30 \mathrm{mg} / \mathrm{kg}$ in vivo); APAP (Acetaminophen: $5 \mathrm{mM}$ in vitro, $1 \mathrm{~g} / \mathrm{kg}$ in vivo); APAP + CAY (combination of Acetaminophen and CAY10591 in the stated doses); APAP + RES (combination of Acetaminophen and Resveratrol in the stated doses). Data are expressed as means \pm SEM $(\mathrm{n}=6): * \mathrm{P}<0.05$, *** $\mathrm{P}<0.001$ vs. respective control.

Effects of CAY in comparison with RES treatment on hepatocyte viability in vitro and relative SIRT1 activity in vitro and in vivo

Measurement of hepatocyte viability (using MTT test) in in vitro experiments and relative SIRT1 activity both in vitro and in vivo are shown in Figure 3. APAP treatment significantly reduced viability $(\mathrm{P}<0.001)$ of cultured hepatocytes in comparison to the untreated control group and the group treated only by RES or CAY. MTT test showed that RES and CAY did not have toxic effect on hepatocytes in cell culture and that CAY more 
potently increased APAP-reduced hepatocyte viability (Fig. 3a). APAP also markedly reduced SIRT1 enzyme activity (by $34 \%$ in vitro and $20 \%$ in vivo) as shown in Figure 3b. Furthermore, RES and especially CAY increased SIRT1 activity compared to control and APAP treatments. The same trend was observed as in vitro as in vivo. In summary, the relationship between activity and viability was indirectly proportional.
Effect of RES, CAY and APAP on SIRT1 expression levels in rats

In comparison with SIRT1 activity mentioned above (Fig. 3b), there were no significant changes on the SIRT1 expression in Western blot analysis (Fig. 4). According to our analysis, single dose treatment with APAP, RES and CAY had no effect on the total endogenous amount of SIRT1 expression.

a)

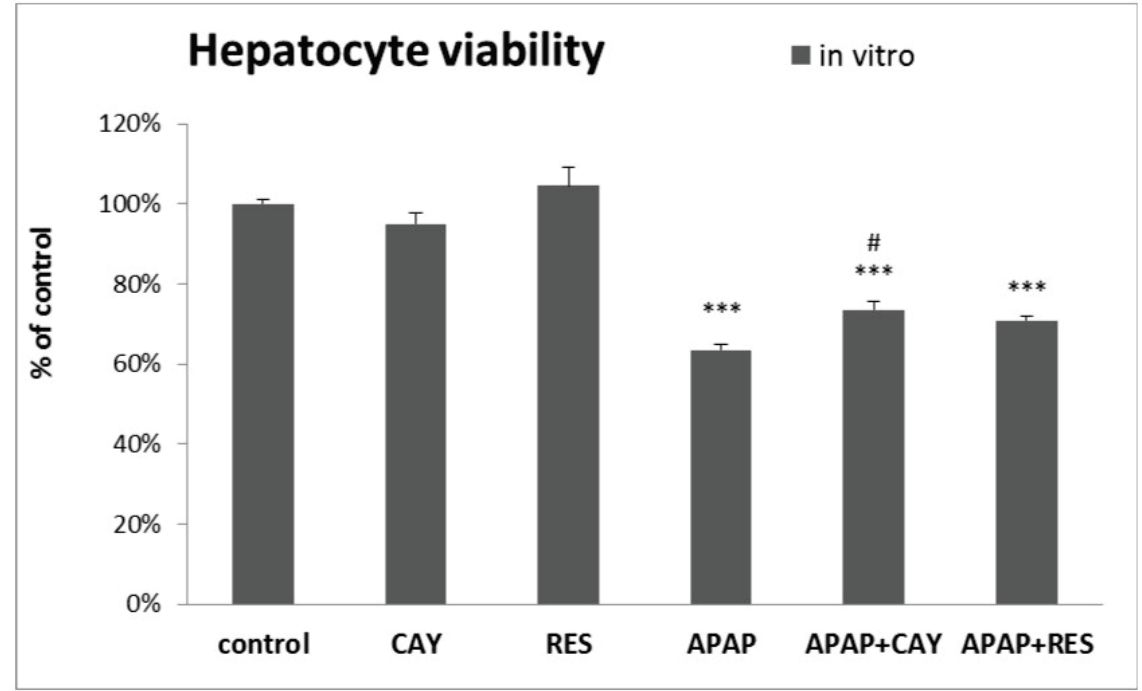

b)

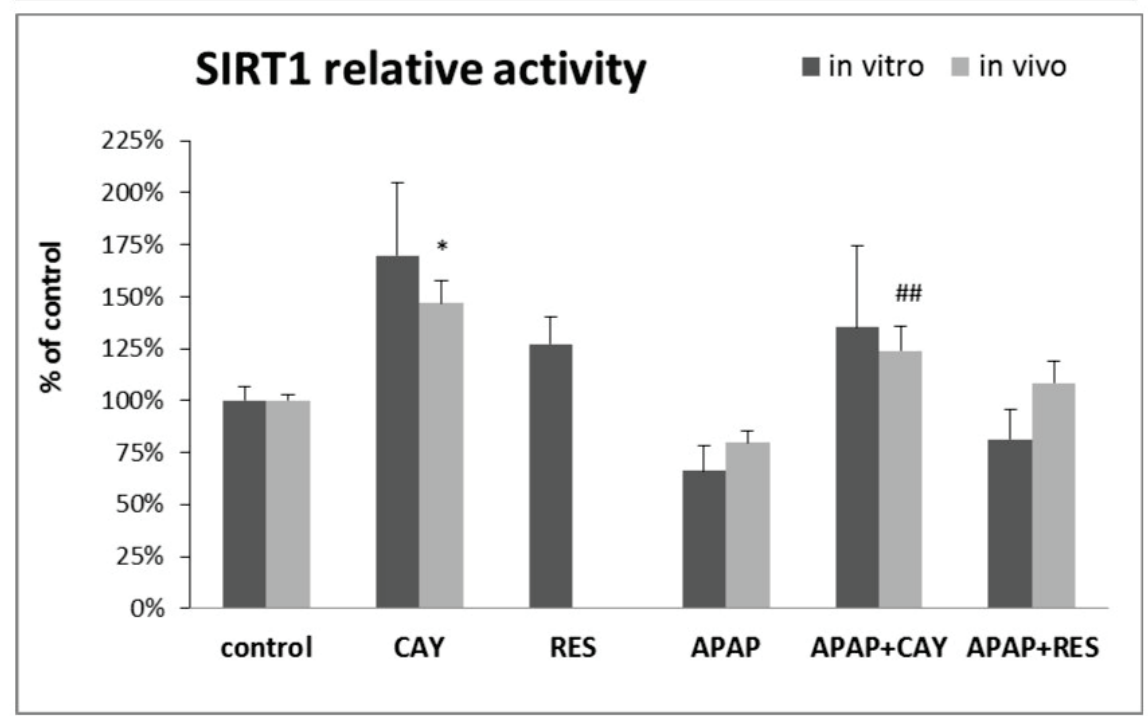

Fig. 3. Effect of specific SIRT1 activator, CAY10591, in comparison with resveratrol treatment in APAP-induced hepatocyte/liver injury on a) Hepatocyte viability in in vitro experiments and b) SIRT1 relative activity in in vitro and in vivo experiments. Control (24 hourvehicle treated hepatocytes or rats); CAY (CAY10591: $30 \mu \mathrm{M}$ in vitro, $0.5 \mathrm{mg} / \mathrm{kg}$ in vivo); RES (Resveratrol: $20 \mu \mathrm{M}$ in vitro, $30 \mathrm{mg} / \mathrm{kg}$ in vivo); APAP (Acetaminophen: $5 \mathrm{mM}$ in vitro, $1 \mathrm{~g} / \mathrm{kg}$ in vivo); APAP + CAY (combination of Acetaminophen and CAY10591 in the stated doses); APAP + RES (combination of Acetaminophen and Resveratrol in the stated doses). Data are expressed as means \pm SEM ( $\mathrm{n}=6$ for MTT test and in vivo SIRT1 activity, $\mathrm{n}=3$ for in vitro SIRT1 activity): $* \mathrm{P}<0.05, * * * \mathrm{P}<0.001$ vs. respective control; \# $\mathrm{P}<0.05$, \#\# $\mathrm{P}<0.01$ vs. respective APAP group.

\section{Histological observations}

The general structure of the liver parenchyma of all rats was well-preserved. Liver trabecules were arranged radially around the central vein and portal spaces were well visible (Fig. 5). No signs of steatosis, inflammation (except of rare small mononuclear infiltrates), hepatocellular necrosis or fibrosis were observed in the rat liver of control (Fig. 5a) and CAY 
(Fig. 5b). However, there were visible several mitotic hepatocytes in otherwise normal liver parenchyma of these rats, especially in CAY treated ones (Fig. 5c). Histological changes in the liver induced by APAP were not significant (Fig. 5d). APAP caused slight increase in the appearance and number of apoptotic hepatocytes (Fig. 5e) and apoptotic bodies surrounded by a mononuclear infiltrate (Fig. 5f). No mitotic hepatocytes were found in the liver of APAP treated rats. Liver parenchyma had normal morphology after application of APAP followed by CAY (Fig. 5g) or resveratrol (Fig. 5h) to rats.

a)

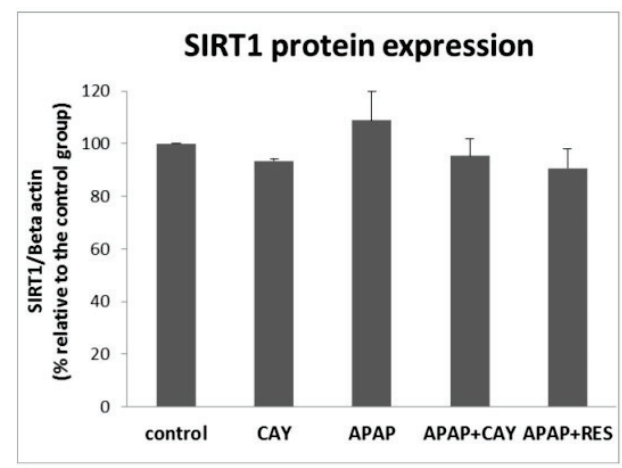

b)

SIRT1

Beta-actin

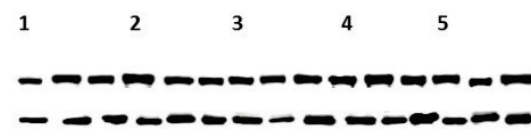

Fig. 4. Effect of specific SIRT1 activator, CAY10591, in comparison with resveratrol treatment in APAP-induced liver injury in vivo on a) quantification of SIRT1 expression levels by densitometry. The intensity of each panel was normalized to the intensity of corresponding beta-actin band. Control ( 24 hour-vehicle treated rats); CAY (CAY10591: $0.5 \mathrm{mg} / \mathrm{kg}$ ); APAP (Acetaminophen: $1 \mathrm{~g} / \mathrm{kg}$ ); APAP + CAY (combination of Acetaminophen and CAY10591 in the stated doses); APAP + RES (combination of Acetaminophen $1 \mathrm{~g} / \mathrm{kg}$ and Resveratrol $30 \mathrm{mg} / \mathrm{kg}$ ). Data are expressed as means $\pm \operatorname{SEM}(n=3)$. b) Western blot images are shown as three samples of each treated group: 1. Control, 2. CAY, 3. APAP, 4. APAP+CAY, 5. APAP+RES.

\section{Discussion}

Our and other previous studies demonstrated that small polyphenolic molecules with antioxidant properties such as resveratrol reduced parameters of liver injury in fulminant hepatic failure induced by LPS/D-GAlN combination (Farghali et al. 2014, Kemelo et al. 2014, Lekić et al. 2013). We chose acute rat APAP intoxication followed by potentially hepatoprotective compounds RES and CAY for our present study. The liver impairment was much lower after APAP treatment than after
LPS/D-GAIN and did not lead to fulminant hepatic failure. We used this model of mild hepatic impairment because it more resembles the human APAP-induced liver injury with potentially following pharmacological intervention. Despite it was shown that rats are resistant to APAP hepatotoxicity in compare to mice (hepatotoxic dose is $400-600 \mathrm{mg} / \mathrm{kg}$ for mice with contrast of $1-2 \mathrm{~g} / \mathrm{kg}$ for rats) (McGill et al. 2012), some recent publications found that human hepatocytes are much more resistant to APAP toxicity than hepatocytes originated from other species (Jemnitz et al. 2008). Many factors (e.g. interspecies variability in expression and/or activity of metabolizing enzymes and membrane transporters, formation of NAPQI adducts, oxidative stress, disruption of mitochondrial function, protein nitrosylation, DNA damage among others) contribute to processes leading to APAP-induced liver damage. The electrophilic metabolite NAPQI, which is mainly produced by CYP2E1, is conventionally thought to initiate the toxic processes in liver. Surprisingly, some of CYP2E1 activity data do not support that only formation of reactive metabolite alone can explain interspecies variability after APAP intoxication (Jemnitz et al. 2015). Moreover, liver regeneration, which is final outcome of APAP-induced sublethal liver injury, begins at about $12 \mathrm{~h}$ and continue until about $72 \mathrm{~h}$ post-dosing and can thus attenuate the final biochemical and histological parameters of hepatotoxicity evaluated from samples collected on a single late time point (e.g. 24 h) (Jaeschke et al. 2013).

In the present study, APAP significantly increased ALT and total bilirubin (specific markers of liver injury) levels with the same trend in vivo as in vitro. RES and CAY were administrated after pretreatment of APAP, and both RES and CAY significantly reduced ALT and also total bilirubin levels in in vivo experiments and enhanced APAP-reduced hepatocyte viability in vitro. As expected and in accordance with other studies (Kučera et al. 2012, McGill et al. 2012), we did not observe marked hepatocyte necrosis in liver of APAP treated rats as evidenced from histological findings. Interestingly, there were visible several mitotic hepatocytes in otherwise normal liver parenchyma of CAY treated rats. It can be explained by induction of hepatocyte proliferation due to increased SIRT1 activation leading to regulation SIRT1/p53 signaling pathway (Braidy et al. 2011, Hwang et al. 2013, Jaeschke et al. 2013, Wang et al. 2015). 

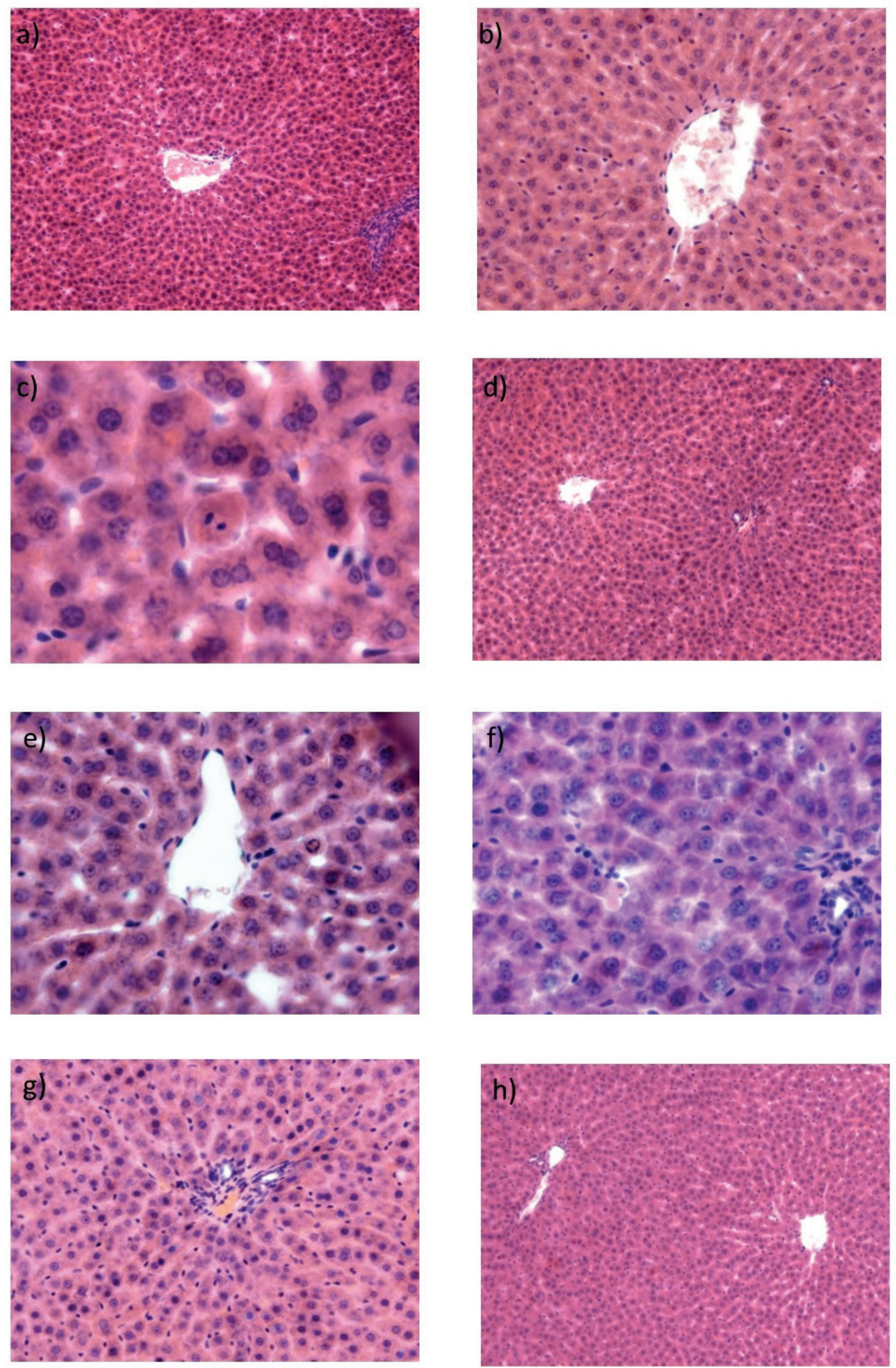

Fig. 5. Representative histopathological samples of livers taken from (a) control rats and animals treated with (b,c) CAY (CAY10591); $(\mathbf{d}, \mathbf{e}, \mathbf{f})$ APAP (Acetaminophen); (g) APAP + CAY; and (h) APAP + RES (combination of Acetaminophen and Resveratrol) for $24 \mathrm{~h}$. Hematoxylin and eosin (magnification $\times 200$ with a detailed view in $c, e, f)$. 
In the case of RES, its hepatoprotetive effect may be due to its well-known antioxidant effects. Moreover, Wang et al. (2015) have found that repeated RES pretreatment of mice significantly increased SIRT1 protein expression and prevented APAP-induced hepatotoxicity revealed as decreased overall necrosis, possibly by inhibition of CYP-mediated APAP bioactivation, and regulation of SIRT1/p53 signaling pathway. RES can by this way promote hepatocyte proliferation and thus facilitate liver regeneration after APAP-induced liver injury. In the case of CAY, which is a selective activator of SIRT1, it can be hypothesized that its hepatoprotective effect may be due to the same SIRT1/p53 signaling pathway as RES. In contrast with study of Wang et al. (2015), there were no significant changes on the SIRT1 expression in Western blot analysis. This difference can be explained by only one application of lower dose $(30 \mathrm{mg} / \mathrm{kg})$ of RES in our experimental settings instead of several day lasting repeated high dose RES pretreatments in the study of Wang et al. (2015) that could induce SIRT expression. However, the SIRT1 activity was highly increased by RES and CAY addition to APAP both in vivo and in vitro that was accompanied by reduction of oxidative stress markers and hepatocyte/liver injury in our study. Furthermore, APAP reduced SIRT1 activity was accompanied simultaneous enhanced oxidative damage as evidences from increased TBARS, $\mathrm{CD}$, and $\mathrm{NO}_{2}^{-}$levels and pronounced liver and hepatocyte injury both in vivo and in vitro. Indeed, some recent studies have shown that SIRT1 is a redox sensitive molecule that can be inactivated by oxidative stress (Braidy et al. 2011, Hwang et al. 2013).

SIRT1 itself has multiple biological activities including gene silencing, stress resistance, apoptosis, regulation of cell proliferation, aging, inflammation, and acts as molecular sensor of nutritional status and protects against oxidative stress (Hwang et al. 2013, Kutinová Canová et al. 2011). However, it should be kept in mind that SIRT1 expression and activity can be modulated at different levels. It was shown that the age-related increasing SIRT1 expression in rat liver and other organs is accompanied by a simultaneous reduction of SIRT1 activity (Braidy et al. 2011). Moreover, RES is thought to activate SIRT1 allosterically (Farghali et al. 2013). It binds to the non-catalytic N-terminus of SIRT1 to cause a conformational change that lowers its Michaelis constant (Howitz et al. 2003). SIRT1 in turn deacetylates and suppresses transcription factors such as NF- $\kappa B$ responsible for induction of proinflammatory cytokines and pro-apoptotic factors (Yeung et al. 2004, Kemelo et al. 2014). Moreover, SIRT1 is also involved in regulation of SIRT1/p53 and other antiapoptotic (e.g. SIRT1/p66shc) signaling pathways (Shan et al. 2015, Wang et al. 2015). It can explain the almost complete absence of apoptotic hepatocytes in liver tissue sections of RES+APAP and CAY+APAP treated rats connected with enhanced SIRT activity unlike APAP alone.

In conclusion, resveratrol and CAY attenuated APAP-induced hepatotoxicity in vivo and in vitro in our study. Moreover, both drugs enhanced APAP-reduced SIRT1 activity but not SIRT1 expression. Therefore, our results show that modulation of the SIRT1 activity plays a role in hepatoprotection. To better understand the role of SIRT1 in hepatoprotection helped us novel specific SIRT1 activator CAY10591. Several other small molecules are being synthesized with higher pharmacologic potency compared with the polyphenolic SIRT1 activator resveratrol. In addition, class III histone deacetylases (sirtuins) are becoming increasingly recognized as important epigenetic drug targets in various diseases (Huber et al. 2011) thus suggesting modulation of sirtuin activity could provide an interesting and novel therapeutic option.

\section{Conflict of Interest}

There is no conflict of interest.

\section{Acknowledgements}

The authors would like to thank Libuse Slehobrova and Marcela Blazkeova for their skilful technical assistance. This work was supported by the institutional program PRVOUK-P25/LF1/2 and research grant GAUK 916314.

\section{References}

BAJT ML, COVER C, LEMASTERS JJ, JAESCHKE H: Nuclear translocation of endonuclease G and apoptosisinducing factor during acetaminophen-induced liver cell injury. Toxicol Sci 94: 217-225, 2006.

BAUR JA: Biochemical effects of SIRT1 activators. Biochim Biophys Acta 1804: 1626-1634, 2010. 
BERRY MN, EDWARDS AM, BARRIT JG: Isolated Hepatocytes Preparation, Properties and Applications. Elsevier, Amsterdam, 1991.

BISHAYEE A, DARVESH AS, POLITIS T, MCGORY R: Resveratrol and liver disease: from bench to bedside and community. Liver Int 30: 1103-1114, 2010.

BRAIDY N, GUILLEMIN GJ, MANSOUR H, CHAN-LING T, POLJAK A, GRANT R: Age-related changes in $\mathrm{NAD}^{+}$metabolism oxidative stress and Sirtl activity in Wistar rats. PLoS One 6: e19194, 2011.

BRUNE K, RENNER B, TIEGS G: Acetaminophen/paracetamol: a history of errors, failors and faile decisions. Eur $J$ Pain 19: 953-963, 2015.

CHANG H-C, GUARENTE L: SIRT1 and other sirtuins in metabolism. Trends Endocrinol Metab 25: 138-145, 2014.

ČERNÝ D, KUTINOVÁ CANOVÁ N, MARTINEK J, HOŘÍNEK A, KMONÍČKOVÁ E, ZIDEK Z, FARGHALI H: Effects of resveratrol pretreatment on tert-butylhydroperoxide induced hepatocyte toxicity in immobilized perifused hepatocytes: Involvement of inducible nitric oxide synthase and hemoxygenase-1. Nitric Oxide 20: $1-8,2009$.

DU K, MCGILL MR, XIE Y, BAJT ML, JAESCHKE H: Resveratrol prevents protein nitration and release of endonucleases from mitochondria during acetaminophen hepatotoxicity. Food Chem Toxicol 81: 62-70, 2015.

FARGHALI H, ČERNÝ D, KAMENÍKOVÁ L, MARTÍNEK J, HOŘÍNEK A, KMONÍČKOVÁ E, ZÍDEK Z: Resveratrol attenuates lipopolysaccharide-induced hepatitis in D-galactosamine sensitized rats: role of nitric oxide synthase-2 and heme oxygenase-1. Nitric Oxide 21: 216-225, 2009.

FARGHALI H, KUTINOVÁ CANOVÁ N, LEKIC N: Resveratrol and related compounds as antioxidants with an allosteric mechanism of action in epigenetic drug targets. Physiol Res 62: 1-13, 2013.

FARGHALI H, KUTINOVÁ CANOVÁ N, ZAKHARI S: Hepatoprotective properties of extensively studied medicinal plant active constituents: possible common mechanisms. Pharmaceut Biol 53: 1-11, 2014.

FEIGE JN, LAGOUGE M, CANTO C, STREHLE A, HOUTEN SM, MILNE JC, LAMBERT PD, MATAKI C, ELLIOTT PJ, AUWERX J: Specific SIRT1 activation mimics low energy levels and protects against dietinduced metabolic disorders by enhancing fat oxidation, Cell Metab 8: 347-358, 2008.

HOWITZ KT, BITTERMAN KJ, COHEN HY, LAMMING DW, LAVU S, WOOD JG, ZIPKIN RE, CHUNG P, KISIELEWSKI A, ZHANG LL, SCHERER B, SINCLAIR DA: Small molecule activators of sirtuins extend Saccharomycetes cerevisiae lifespan. Nature 425: 191-196, 2003.

HUBER K, SUPERTI-FURGA G: After the grape rush: Sirtuins as epigenetic drug targets in neurodegenerative disorders. Bioorg Med Chem 19: 3616-3624, 2011.

HWANG JW, HONGWEI Y, CAITO S, SUNDAR IK, RAHMAN I: Redox regulation of SIRT1 in inflammation and cellular senescence. Free Radic Biol Med 61: 95-110, 2013.

HY, NI B, ENTZEROTH M, STUNKEL W: 12SIRT1 modulating compounds from high-throughput screening as antiinflammatory and insulin-sensitizing agents to study biological effects of stimulated SIRT1 enzyme. J Biomol Screen 11: 959-967, 2006.

INGAWALE DK, MANDLIK KM, NAIK SR: Models of hepatotoxicity and the underlying cellular, biochemical and immunological mechanism(s): a critical discussion. Enviroment Toxicol Pharmacol 37: 118-133, 2014.

JAESCHKE H, WILIAMS CD, MCGILL MR, XIE Y, RAMACHANDRAN A: Models of drug-induced liver injury for evaluation of phytotherapeutics and other natural products. Food Chem Toxicol 55: 279-289, 2013.

JEMNITZ K, VERES Z, MONOSTORY K, KOBORI L, VERECZKEY L: Interspecies differences in acetaminophen sensitivity of human, rat, and mouse primary hepatocytes. Toxicol In Vitro 22: 961-967, 2008.

KAPLOWITZ N: Acetaminophen hepatoxicity: what do we know, what don't we know, and what do we do next? Hepatology 40: 23-26, 2004.

KARTHIVASHAN G, ARUSELVAN P, FAKURAZI S: Pathways involved in acetaminophen hepatotoxicity with specific targets for inhibition/downregulation. RSC Adv 5: 62040-62051, 2015.

KEMELO MK, WOJNAROVÁ L, KUTINOVÁ CANOVÁ N, FARGHALI H: D-galactosamine/lipopolysaccharideinduced hepatotoxicity downregulates sirtuin 1 in rat liver: role of sirtuin 1 modulation in hepatoprotection, Physiol Res 63: 615-623, 2014. 
KUČERA O, ROUŠAR T, STAŇKOVÁ P, HAŇÁČKOVÁ L, LOTKOVÁ H, PODHOLA M, ČERVINKOVÁ Z: Susceptibility of rat non-alcoholic fatty liver to the acute toxic effect of acetaminophen. $J$ Gastroenterol Hepatol 27: 323-330, 2012.

KUTINOVÁ CANOVÁ N, MARTINEK J, KMONIČKOVÁ E: Modulation of spontaneous and lipopolysaccharideinduced nitric oxide production and apoptosis by D-galactosamine in rat hepatocyte culture: the significance of combinations of different methods. Toxicol Mech Methods 18: 63-74, 2008.

KUTINOVÁ CANOVÁ N, GAIER N, FARGHALI H: Perspectives on pharmacological and clinical benefits from sirtuin 1 activators in oxidative damage (in Czech). Cas Lek Cesk 151: 187-189, 2012.

LEKIC N, KUTINOVÁ CANOVÁ N, HOŘÍNEK A, FARGHALI H: The involvement of heme oxygenase 1 but not nitric oxide synthase 2 in a hepatoprotective action of quercetin in lipopolysaccharide-induced hepatotoxicity of D-galactosamine sensitized rats. Filoterapia 87: 20-26, 2013.

MCGILL MR, WILLIAMS CD, XIE Y, RAMACHADRAN A, JAESCHKE H: Acetaminophen-induced liver injury in rats and mice: Comparison of protein adducts, mitochondrial dysfunction, and oxidative stress in the mechanism of toxicity. Toxicol App Pharmacol 264: 387-394, 2012.

NAYAGAM VM, WANG X, TAN YC, POULSEN A, GOH KC, NG T, WANG H, SONG YEUNG F, HOBERG JE, RAMSEY CS, KELLER MD, JONES DR, FRYE RA, MAYO MW: Modulation of NF-kB-dependent transcription and cell survival by the SIRT1 deacetylase. EMBO J 23: 2369-2380, 2004.

ROUŠAR T, PǍ̌IIK P, KUČERA O, BARTOŠ M, ČERVINKOVÁ Z: Glutathione reductase is inhibited by acetaminophen-glutathione conjugate in vitro. Physiol Res 59: 225-232, 2010.

RUDERMAN NB, XU XJ, NELSON L, CACICEDO JM, SAHA AK, LAN F, IDO Y: AMPK and SIRT1: a longstanding partnership? Am J Physiol Endocrinol Metab 298: E751-E760, 2010.

SHAN W, GAO L, ZENG W, HU Y, WANG G, LI M, ZHOU J, MA X, TIAN X, YAO J: Activation of the SIRT1/p66shc antiapoptosis pathway via carnosic acid-induced inhibition of miR-34a protects rats against nonalcoholic fatty liver disease. Cell Death Dis 6: e1833, 2015.

SILVA JP, WAHLESTEDT C: Role of Sirtuin 1 in metabolic regulation. Drug Discov Today 15: 781-791, 2010.

WANG Z, JIANG Y, FAN X, TAN H, ZENG H, WANG Y, CHEN P, HUANG M, BI H: Hepatoprotective effect of resveratrol against acetaminophen-induced liver injury is associated with inhibition of CYP-mediated bioactivation and regulation of SIRT-p53 signaling pathways. Toxicol Lett 236: 82-89, 2015.

YANG SR, WRIGHT J, BAUTER M, SEWERYNIAK, KODE A, RAHMAN I: Sirtuin regulates cigarette smokeinduced proinflammatory mediator release via RelA/p65 NF-kappaB in macrophages in vitro and in rat lungs in vivo: implications for chronic inflammation and aging. Am J Physiol Lung Cell Mol Physiol 292: L567L576, 2007. 